\title{
Aves asociadas al Aeropuerto Carriel Sur de Talcahuano, sur de Chile: evaluación de peligro aviario
}

\section{Associated birds to the Carriel Sur Airport of Talcahuano, south of Chile: evaluation of endangered aviary}

\author{
Carlos Barrientos ${ }^{1}$, Daniel González-Acuña ${ }^{1 *}$, Lucila Moreno ${ }^{2}$, Karen Ardiles $^{1}$ \& Ricardo A. \\ FIGUEROA R. ${ }^{3}$ \\ ${ }^{1}$ Facultad de Ciencias Veterinarias, Universidad de Concepción, Departamento de Ciencias Pecuarias, Casilla 537, Chillán, \\ Chile. \\ ${ }^{2}$ Facultad de Ciencias Naturales y Oceanográficas, Universidad de Concepción, Departamento de Zoología, Casilla 160-C, \\ Concepción, Chile. \\ ${ }^{3}$ Escuela de Graduados, Facultad de Ciencias Forestales y Recursos Naturales, Universidad Austral de Chile, Valdivia, Chile. \\ *E-mail: danigonz@udec.cl
}

\section{RESUMEN}

Entre el verano de 2002 y 2005 se evaluó la riqueza y abundancia de aves asociadas al Aeropuerto Carriel Sur de Talcahuano y se estimó el riesgo de colisión que representaría cada especie para la aeronavegación. Las aves fueron contabilizadas en 10 puntos de conteos distribuidos en distintos hábitats alrededor del las pistas de aterrizaje. El riesgo de colisión fue estimado mediante el índice de peligro aviario (IPA) el cual combina tamaño, abundancia e historial de colisiones de cada especie. Durante el periodo de estudio se registraron 63 especies, mas 10 especies que fueron registradas después del verano de 2005. Las especies más abundantes fueron el chirigüe, la gaviota dominicana, el queltehue y la loica (28\%, $14 \%, 10 \%, 8 \%$ del total de registros individuales). Con excepción de la gaviota dominicana, todas las especies exhibieron ciclos estacionales de abundancia. La gaviota dominicana mostró irrupciones multianuales aparentemente por factores ambientales no bien determinados. Las especies que representarían un mayor riesgo de colisión para la aeronavegación son la gaviota dominicana (IPA = 36), el queltehue (IPA = 24), el gaviotín sudamericano (IPA = 16), el jote de cabeza negra $($ IPA $=12)$, el yeco $(I P A=9)$ y el tiuque (IPA = 8). Sin embargo, el IPA de cada especie varió temporalmente desde valores muy altos a valores muy bajos según los cambios de abundancia. Este estudio refleja la necesidad de evaluaciones de largo plazo de los cambios en el tamaño poblacional de especies de aves para una mejor aproximación del riesgo de colisiones aves-aeronaves.

Palabras claves: Abundancia, avifauna, colisión, Concepción, diversidad.

\begin{abstract}
Between the summer 2002 and 2005 was evaluated the richness and abundance of birds associated to the Carriel Sur Airport of Talcahuano and was estimated the collision risk representing each species for air navigation. Birds were counted in 10 count points distributed in different habitats around the landing runways. The collision risk was estimated by the Avian Hazard Index (AHI) which combines size, abundance and history of collisions of each species. During the study period 63 species were recorded, plus 10 aditional species were recorded after summer 2005. The most abundant species were the Grassland Yellow-Finch, Kelp Gull, Southern Lapwing Long-tailed Meadowlark (28\%, 14\%, 10\%, 8\% of all individual records). Excepting Kelp Gull, all remaining species exhibited seasonal cycles of abundance. The Kelp Gull showed multiyear outbreaks triggered. The species representing a higher collision risk for air navigation are the Kelp Gull (API = 36), Southern Lapwing $(\mathrm{AHI}=24)$, South American Tern $(\mathrm{AHI}=16)$, Black Vulture $(\mathrm{AHI}=12)$, Neotropic Cormorant $(\mathrm{AHI}=9)$ and Chimango Caracara $(\mathrm{AHI}=8)$. However, the each species' AHI varied temporally from very high to very low values according to changes in abundance. This study reflects the need for long-term assessments of changes in population size of bird species to better approximate the risk of bird-aircraft collisions.
\end{abstract}

KeYwords: Abundance, bird species, collision, Concepción, diversity. 


\section{INTRODUCCIÓN}

Cuando aves y aeronaves utilizan un mismo espacio aéreo existe un alta probabilidad de colisión entre ellas (Sodhi 2002, Cleary \& Dolbeer 2005). Esto se conoce en aeronavegación como peligro aviario y representa un problema serio para el tráfico aéreo a escala mundial (Magalhães 2000, Martelli 2000, Matijaca 2000). En las últimas décadas, el desarrollo de aeronaves de alta velocidad y menos ruidosas parece haber acentuado el problema ya que las aves no alcanzan a advertir su presencia ni a evadir el impacto (Dekker 2000, Matijaca 2000, Olivares 1995). En su nivel más grave, las colisiones con aves pueden causar pérdidas económicas sustanciales, muertes de pasajeros y/o pérdida de carga (Allan 2000, Thorpe 2003, Bitebekezi 2007). Por está razón, las colisiones aves-aeronaves son reconocidos como un asunto prioritario por las autoridades aeronáuticas y ha obligado a desarrollar protocolos para reducir el riesgo de accidentes (Dekker 2000, Thorpe 2003).

Paradojalmente, la mayor parte de los aeropuertos están localizados en áreas de concentración de fauna silvestre. Por razones de seguridad, los terminales aéreos son establecidos relativamente lejos de centros urbanos, y de ahí, en sitios donde existen condiciones ambientales favorables para las aves silvestres (e.g., praderas abandonadas y sistemas lacustres [Caccamise et al. 1996, Bitebekezi 2007, Garland et al. 2009]). Además, la ubicación de los aeropuertos usualmente coincide con aquella de los vertederos sanitarios los cuales atraen a muchas especies de aves carroñeras que vuelan a gran altura (Caccamise et al. 1996, Dolbeer 2006). Por otra parte, la exclusión de terrenos alrededor de las pistas de aterrizaje facilita el crecimiento de la vegetación natural la cual proporciona sitios apropiados para el refugio, alimentación y nidificación para la fauna silvestre, particularmente aves (Baker \& Brooks 1981, Dolbeer et al. 2000, Witmer \& Fantinato 2003). Todos estos factores combinados pueden aumentar considerablemente el riesgo de colisiones en el área de influencia de los aeropuertos.

La situación en los aeropuertos nacionales no es distinta de lo que ocurre a nivel internacional. El incremento del flujo aéreo y la gran cantidad de aves residentes parece ser la causa del aumento de los reportes de colisiones con aves por parte de operarios y pilotos en los aeropuertos chilenos. El Aeropuerto Carriel Sur de Talcahuano (ACST) es precisamente el aeropuerto que presenta la mayor cantidad de colisiones en Chile (Martelli 2000). La Dirección General de Aeronáutica Civil (DGAC) ha notificado 169 incidentes aves-aeronaves en Chile entre 1975-2002 de los cuales el 41,4\% ocurrió en el ACST (Martelli 2000). Del total de las colisiones notificadas entre 1994-2006 en el ACST, 34 ocurrieron en la fase de vuelo de aproximación, 32 en el recorrido de aterrizaje, 21 en recorrido de despegue, dos en ascenso, dos en descenso y una en rodaje ${ }^{1}$.

Un primer paso para evaluar el peligro aviario y formular programas de control de accidentes en los aeropuertos es describir la comunidad de aves que allí habita y monitorear los cambios temporales de sus tamaños poblaciones y sus patrones de actividad diaria (González-Acuña et al. 2006). Aquí, documentamos los resultados de un estudio de mediano plazo (tres años) sobre la comunidad de aves asociada al ACST. Nuestros objetivos fueron: (1) determinar la riqueza y composición de especies, (2) estimar la variación temporal en el número de aves, y (3) evaluar el riesgo potencial de colisión que representa cada especie.

\section{MATERIAL Y MÉTODOS}

Área DE ESTUdio

El ACST está ubicado en la conurbación Concepción-

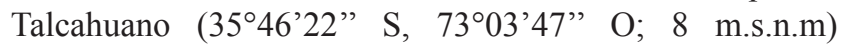
abarcando un área total de 260 ha. La ciudad de Talcahuano se localiza en el borde costero y Concepción a no más de tres kilómetros de este. Dentro del terminal aéreo se sitúan dos pistas de aterrizaje con una orientación norte-sur, teniendo la pista principal una longitud de $2.300 \mathrm{~m}$ y un ancho de 45 m. Dentro de los límites se presentan matorrales abiertos, pastizales agrícolas abandonados, praderas de cultivo y ganaderas, lagunas permanentes y temporales, y prados con pasto corto alrededor de las pistas.

El paisaje que circunda al ACST se caracteriza por contener una alta diversidad vegetacional y orográfica en un espacio relativamente reducido (Martelli 2000). Los elementos más característicos del paisaje dentro del área son desembocaduras de ríos, penínsulas boscosas, asentamientos urbanos, barrios industriales, humedales, bahías, campos agrícolas y áreas de colinas con plantaciones forestales. La desembocadura del río Bío-Bío y Andalíen constituyen zonas de descanso, alimentación y crianza de aves marinas. Las penínsulas boscosas incluyen la península de Hualpén y Tumbes, en donde la vegetación boscosa presente allí es utilizada como refugio de diversas especies de aves tanto terrestres como marinas. Las áreas urbanas incluyen las periurbes de Concepción, Talcahuano, Hualpencillo, San Pedro de la Paz, Hualqui, Chiguayante, Penco, Lirquén y Tomé. Las bahías de Concepción y San Vicente están orientadas fundamentalmente al desarrollo portuario comercial y pesquero. Las praderas abandonadas están constituidas principalmente por especies de pastos y hierbas alóctonos. Entre los humedales destacan el humedal de Lenga y la marisma de Rocuant y este último es hábitat de un gran número de especies de aves dulceacuícolas

${ }^{1}$ Comunicación personal, Mauricio Muñoz jefe S.E.I. Aeropuerto Carriel Sur Talcahuano. 
(Victoriano et al. 2006). Las áreas de cerros presentes en el área representan la continuidad de la Cordillera de la Costa. Alrededor del sitio de estudio, la vegetación boscosa original de esta cordillera ha sido sustituida extensamente por plantaciones forestales, principalmente de pino insigne (Pinus radiata). El clima es templado-húmedo con influencia mediterránea. Las precipitaciones y temperatura media anual son 1.330 milímetros y $13^{\circ} \mathrm{C}$ respectivamente (Di Castri \& Hajek 1976).

\section{DiSEÑO DE MUESTREO}

Conteos. El conteo de aves se realizó entre marzo de 2002 y marzo 2005 en 10 puntos de conteo establecidos a lo largo del cinturón de vegetación que rodea las pistas de aterrizaje (Fig. 1). La distribución de las estaciones de conteo abarcó todos los tipos de hábitats dentro de los límites de ACST, incluyendo arbustos achaparrados (estación 1), un pastizal agrícola abandonado (estación 2 y 3), una pradera con pastizal bajo arbolada (estación 4 y 5), una laguna permanente ( $80 \mathrm{~m}$ al oeste de la estación 5), un pastizal bajo con un rodal de pino insigne (estación 6), una pradera de forrajeo para ganado bovino y equino (estación 7), una zona con vegetación escasa y cercana a una carretera (ruta 154; estación 8), praderas con lagunas invernales (estación 9) y un área edificada que abarcó la villa aeronáutica y el terminal aeronáutico (estación 10). Los puntos de conteo estuvieron separados por una distancia de 200 a $400 \mathrm{~m}$ entre si y en cada uno de ellos se contabilizaron todas las aves vistas y escuchadas en un radio de $200 \mathrm{~m}$ durante $10 \mathrm{~min}$. En cada sesión de conteo se incluyó a todas las aves ya sea posadas, caminando o volando hasta una altura de $500 \mathrm{~m}$ sobre el suelo. Las observaciones se hicieron a ojo desnudo o con el apoyo de binoculares $(12 \times 25)$ cuando fue necesario. Con el fin de uniformar cada conteo en relación a la fase del día, cada sesión de conteo se realizó durante las cuatro horas previas al crepúsculo vespertino. Las sesiones de conteos se hicieron dos veces por mes entre marzo de 2002 y diciembre 2003, y una vez por mes entre enero de 2004 y marzo de 2005. Con propósitos comparativos, los conteos se agruparon de acuerdo a las cuatro estaciones climáticas según la fecha calendario; por lo que el número de conteos por cada estación fue como sigue: uno en el verano de 2002; seis en el otoño, invierno y primavera de 2002; tres en el verano, invierno y primavera de 2003 , y verano y primavera de 2004; dos en el otoño de 2003 e invierno de 2004; y cuatro en el otoño de 2004 y verano de 2005.

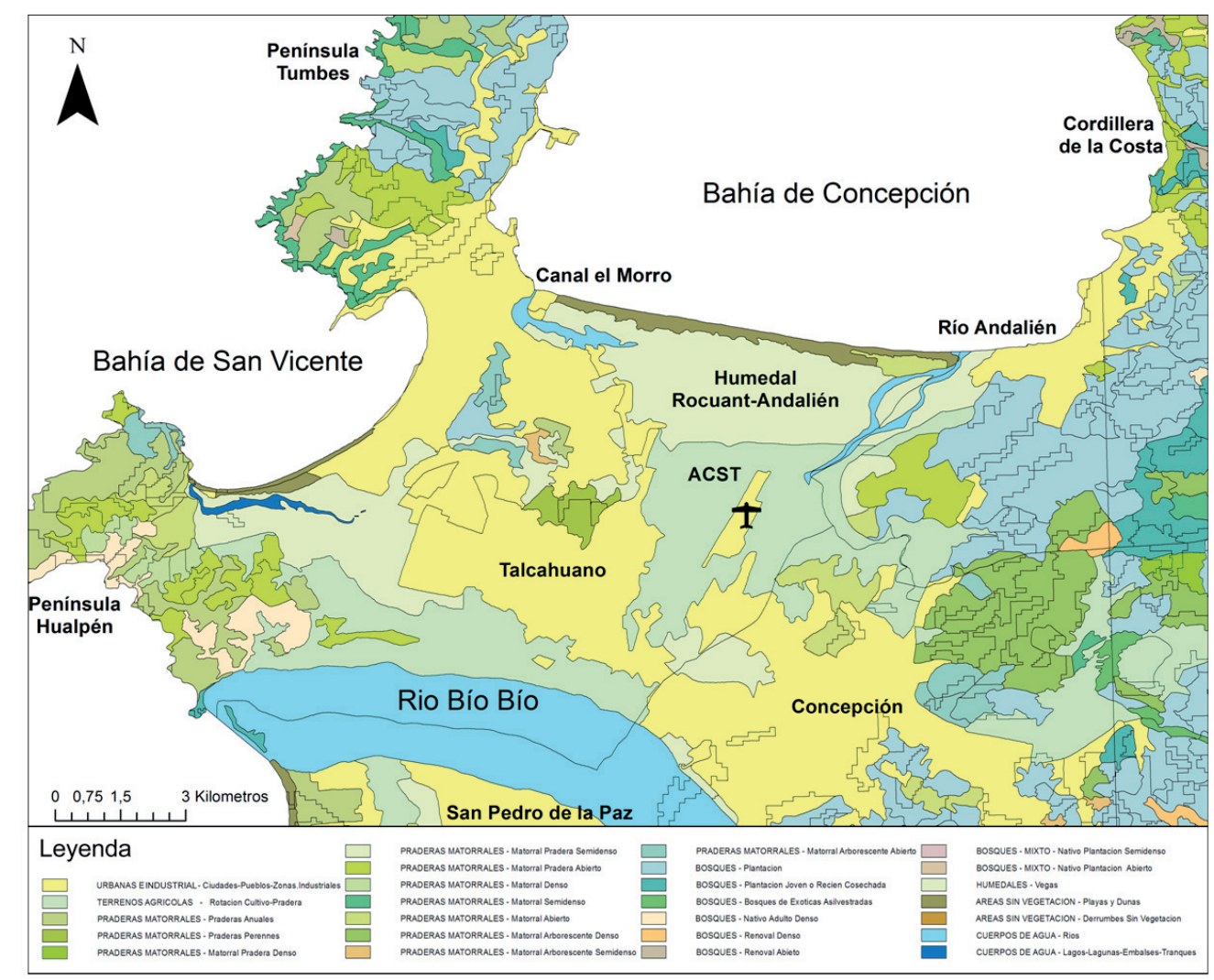

Figura 1. Ubicación del Aeropuerto Carriel Sur de Talcahuano, sur de Chile. En el centro de la figura se muestran las características del paisaje y se indica el uso de suelo en el área.

Figure 1. Location of Carriel Sur Airport of Talcahuano, southern Chile. In the center of the figure the characteristics of the landscape and land uses are shown. 
RiQUEZA Y ABUNDANCIA DE ESPECIES. La riqueza de especies se expresó simplemente como el número de especies detectadas (Rau et al. 1998, Bibby et al. 2000) y se estimó de dos maneras: riqueza bruta y riqueza estandarizada. La primera se refiere al número total de especies registradas combinando todas las estaciones de conteo y todos los conteos estacionales. Sin embargo, debido a que el número de conteos fue desigual entre estaciones climáticas los resultados no son comparables. Así, para hacer comparables los resultados, los valores de riqueza bruta dentro de cada estación climática fueron estandarizados dividiendo a éstos por el número de conteos respectivos. Con similar criterio, la abundancia relativa de cada especie dentro de una estación climática se expresó como el número estandarizado de individuos. El procedimiento fue el siguiente: (i) se sumaron todos los registros de aves individuales incluyendo todas las sesiones y estaciones de conteo, y (ii) los valores totales fueron divididos por el número de sesiones de conteo. Para obtener el promedio del número estandarizado de individuos los valores resultantes fueron divididos por el número de puntos de conteo el cual fue constante en todas las estaciones climáticas. Los rangos de abundancia global se estimaron dividiendo el total de registros individuales estandarizados de cada especie por la suma de los registros individuales estandarizados de todas las especies. Los nombres comunes de las aves se basaron en la Lista Patrón de las Aves Chilenas (Araya et al. 1995) y los nombres científicos se basaron en los criterios de Remsen et al. (2013)

Estimación DE PELIGro AVIARIO. Martelli (2000) desarrolló un índice de peligro aviario (IPA) basado en el peso, participación en colisiones y abundancia de cada especie de ave. Este autor subdividió cada variable en distintas categorías de importancia asignando la categoría 1 al rango de valores más alto, la categoría 2 al siguiente rango más alto, y así sucesivamente. Debido a que el valor del IPA se obtiene por la suma de categorías, los IPA con puntaje más bajo indican un mayor riesgo de colisión. Ya que esto puede causar confusión se decidió modificar el criterio original de Martelli invirtiendo los valores de las categorías y, de ahí, hacer correlativo el valor del IPA con el riesgo de colisión. En este estudio el peso de las aves se dividió en tres categorías: $3=$ alto ( $>1051 \mathrm{~g}$ ó $>71 \mathrm{~cm}$ de longitud corporal), $2=$ medio (96-1050 g o 31-70 cm de longitud total) y $1=$ bajo $(<95 \mathrm{~g}$ o $<30 \mathrm{~cm}$ de longitud corporal). El grado de participación en colisiones se basó sobre la información proporcionada por Martelli (2000) e incluyó las siguientes categorías: $4=$ frecuente $(\geq 5$ casos $), 3=$ menor $(2-4$ casos $), 2=$ puntual $(1$ caso) y $1=$ nula ( $\sin$ reportes). En el caso de la abundancia se asignaron las siguientes categorías basadas en el porcentaje del número estandarizado de individuos: $1=$ muy baja $(<$ $5 \%), 2=$ baja $(5-19 \%), 3=$ media $(20-29 \%), 4=$ alta $(30-$ $49 \%), 5=$ muy alta (50-60\%). Cuando se calculó el IPA global de cada especie se utilizó el número estandarizado de individuos considerando todo el periodo de estudio. Para aquellas especies que alcanzaron los IPA más altos también se estimó la variación estacional de este índice utilizándose el respectivo valor porcentual del número estandarizado de individuos.

AnÁlisis ESTAdísticos. Para determinar si hubouna correlación entre el número de especies registradas y el esfuerzo de muestreo (i.e., sesiones de conteo) se utilizó el coeficiente de correlación no paramétrica de Spearman (Fowler \& Cohen 1986). En el caso de las estimaciones de abundancia, no se consideró estadística inferencial principalmente por tres razones. Primero, la relativa cercanía de las estaciones de conteo y el amplio movimiento de algunas especies pudo no haber asegurado la independencia espacial entre las unidades de muestreo. Debido a que muchos individuos de las especies observadas que no se encontraban posadas en el área de estudio, se encontraban sobrevolando extensamente el sitio de estudio, la abundancia de tales especies posiblemente fue sobreestimada; i.e., los mismos individuos pudieron haber sido contados en más de un punto de conteo dentro de una misma sesión de conteo. Segundo, usualmente los conteos estacionales dentro de un mismo sitio de estudio tampoco son independientes. Finalmente, debido a que nuestro estudio no está basado en un diseño experimental controlado el uso de estadística inferencial es inaplicable. Sin embargo, en muchos casos las diferencias gráficas fueron visualmente evidentes y cualquier prueba de significancia pudo haber sido totalmente innecesaria (e.g. Guthery et al. 2001).

\section{RESULTADOS Y DISCUSIÓN}

RiQueZA DE EsPECIES

En total se registraron 63 especies de aves entre el verano de 2002 y verano de 2005 . Hubo un directa relación entre la riqueza acumulada de especies y el tiempo de monitoreo (Fig. 2). La curva de acumulación se tornó fuertemente asintótica después de un año de monitoreo, pero el 100\% de la especies se detectó solo al final del tercer año de monitoreo. Diez especies adicionales fueron registradas después del verano de 2005. Así, al menos 73 especies pueden ser encontradas en el área de influencia del ACST (ver Anexo 1). Los grupos de aves mejor representados fueron los Passeriformes (30 especies), Charadriiformes (8 especies) y Anseriformes (7 especies; Anexo 1). La riqueza de especies detectada en este estudio supera considerablemente aquella documentada por Martelli (2000) quien registró sólo 33 especies. Además, la riqueza actual de especies en el ACST es proporcionalmente alta comparada con la observada en otros aeropuertos del país tales como el Aeropuerto Presidente Carlos Ibáñez del Campo (ACIC) de Punta Arenas (17 especies, Olivares 1995), Aeropuerto Arturo Merino Benítez (AAMB) de 
Santiago (41 especies, Muñoz 2004) y el Aeródromo Bernardo O`Higgins (ABOC) de Chillán (47 especies, González-Acuña et al. 2006).

La alta riqueza de especies del ACST parece tener una directa relación con la alta diversidad de hábitats en el área. El predominio de especies paserinas se explicaría por la alta disponibilidad de pastizales, juncales y matorrales los cuales aseguran suficiente refugio y alimento (e.g., insectos, semillas) tanto al interior como alrededor de los límites del ACST. Estudios previos en agroecosistemas del sur de Chile han encontrado que estos tipos de hábitats concentran una alta riqueza de aves, incluyendo un gran número de aves paserinas (Estades 1994, Figueroa \& Corales 1999, 2005, Silva-Rodríguez et al. 2008). La existencia de esteros, lagunas naturales, tranques artificiales, marismas, bahías y desembocaduras de ríos dentro del área de influencia de ACST explicaría la presencia del gran número de especies acuáticas (25 especies, Anexo 1). Previamente, GonzálezGajardo et al. (2009) documentaron un alto número de especies dulceacuícolas dentro del área de influencia del ACST. Olivares (1995) argumenta que ríos, riachuelos y charcas explica la presencia de muchas especies de aves acuáticas en el ACIC de Punta Arenas. La amplia diversidad de especies de aves en el ACST atraería también a varias especies de aves rapaces ornitófagas (e.g., cernícalo, peuco, vari, nuco). Además, los pastizales dentro de los aeropuertos pueden sostener una alta densidad de roedores silvestres (Witmer \& Fantinato 2003) y, de ahí, atraer a varias especies de aves rapaces que se alimentan de ellos (ver Anexo 1). De hecho, casi todas las especies de aves rapaces registradas en el sitio de estudio fueron observadas cazando en pastizales

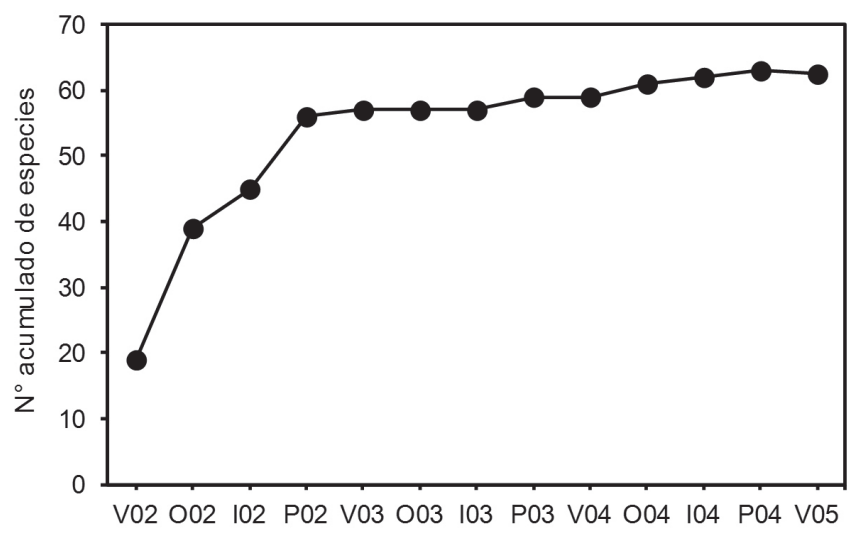

Figura 2. Riqueza acumulada de especies de aves a lo largo del tiempo en el Aeropuerto Carriel Sur de Talcahuano, sur de Chile. $\mathrm{V}=$ verano, $\mathrm{O}=$ otoño, $\mathrm{I}=$ invierno, $\mathrm{P}=$ primavera; los números acompañantes representan los años 2002 a 2005.

FIGURE 2. Accumulated diversity of bird species over time at the Carriel Sur Airport of Talcahuano, southern Chile. V = summer, $\mathrm{O}=$ autumn, $\mathrm{I}=$ winter, $\mathrm{P}=$ spring; the accompanying numbers represent the years 2002 to 2005 . dentro del ACST. Estudios previos sobre la dieta de peucos y milanos bailarines han demostrado que éstos consumen roedores silvestres todo el año (Figueroa \& GonzálezAcuña 2006; González-Acuña et al. 2009). Algunos grupos de aves acuáticas tales como taguas y patos constituyen también una parte importante de la dieta del peuco en el ACST (Figueroa \& González-Acuña 2006). La presencia de grupos de aves carroñeras tales como jotes y gaviotas se debería a la existencia de acumulaciones de desechos orgánicos provenientes de la industria pesquera alrededor del área de influencia del ACST.

El número de especies detectadas en cada estación climática se correlacionó positiva y significativamente con el número de sesiones de conteo $\left(\mathrm{r}_{\mathrm{s}}=0,6, P=0,02, \mathrm{~N}=13\right)$. Esto confirma nuestra presunción que el desbalance estacional en el número de sesiones de conteo afectó las estimaciones de riqueza de especies. En el caso de la riqueza estandarizada, no hubo una tendencia clara en la variación temporal de especies, pudiendo registrarse más especies en distintas estaciones climáticas en años distintos (Fig. 3). Esto podría explicarse ya sea por variaciones ambientales temporales o por problemas de detectabilidad. Debido a falta de datos de pluviometría en el área, no fue evaluado directamente la relación entre lluvias y abundancia de algunas aves, sin embargo es posible que en los inviernos más lluviosos se hayan formado más lagunas o charcas temporales y así más especies dulceacuícolas pudieron ser atraídas al ACST. Algo similar pudo ocurrir en las primaveras o los veranos más lluviosos. La emergencia de larvas de insectos y lombrices sobre las praderas inmediatamente después de las lluvias es un fuerte atrayente para varias aves insectívoras (e.g., zorzal, queltehue). Por otra parte, en primavera y verano cuando las aves inician su periodo reproductivo éstas se vuelven más sigilosas restringiendo su actividad a los sitios de nidificación. Esto también pudo haber causado que algunas especies incluso abandonaran el aeropuerto en busca de sitios seguros para reproducirse. De esta manera, la probabilidad de detectarlas pudo disminuir considerablemente. Independiente de los resultados, se debe tener en cuenta dos importantes consideraciones: (i) la estimación apropiada de la riqueza de especies en un sitio de interés - en este caso un aeropuerto- requiere de un monitoreo de mediano a largo plazo, y (ii) el esfuerzo de muestreo entre estaciones climáticas debe ser balanceado para evitar sesgos temporales y, de ahí, hacer que los resultados sean directamente comparables.

\section{Abundancia Relativa}

En términos globales, la especie más abundante fue el chirigüe seguido de la gaviota dominicana, el queltehue y la loica (Fig. 4). Sin embargo, nótese que el chirigüe dobla o triplica en abundancia a las otras tres especies más abundantes. Los individuos de esta especie de paseriforme 
se reúnen en grandes bandadas durante los meses de otoño e invierno desplazándose activamente por los campos abiertos (Couve \& Vidal 2003, Martínez \& González 2005). Aunque esta conducta pudo haber influenciado positivamente su detectabilidad, el chirigüe es una especie típicamente numerosa en el sur de Chile (Figueroa \& Corales 1999; Figueroa \& Quintana 2001, Silva-Rodríguez et al. 2008). Nótese que entre todas las especies más abundantes (Fig. 4) se encuentran especies típicas de praderas y de ambientes acuáticos. Además, es destacable la presencia de especies carroñeras de gran tamaño tales como el jote de cabeza negra y la gaviota dominicana. Esta combinación de especies con muy distintos hábitos y uso de hábitats refleja claramente las características ambientales y ecológicas en el área de influencia del ACST.

Resultados similares a los obtenidos en el ACST fueron encontrados en el ABOC. En este último sitio, chirigüe, queltehue, loica y tiuque estuvieron entre las especies
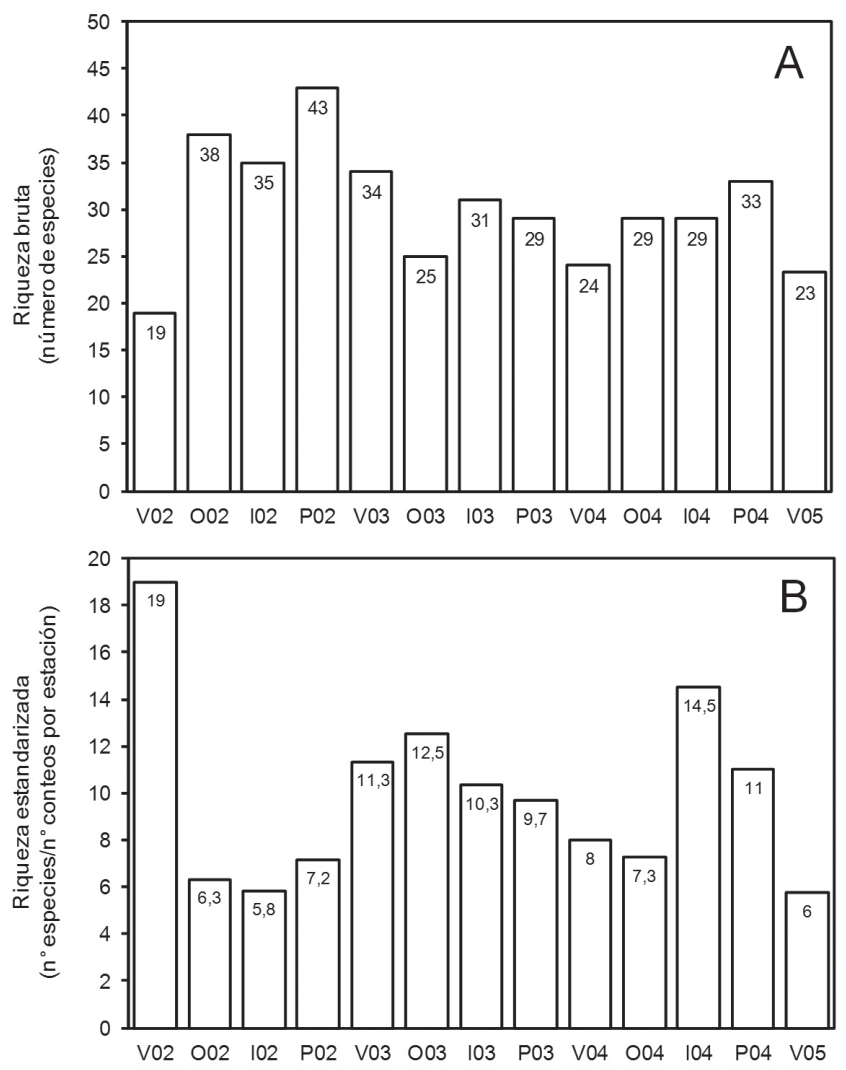

Figura 3. Variación temporal en la riqueza de especies de aves entre 2002 y 2005 en el Aeropuerto Carriel Sur de Talcahuano, sur de Chile. $\mathrm{V}=$ verano, $\mathrm{O}=$ otoño, $\mathrm{I}=$ invierno, $\mathrm{P}=$ primavera; los números acompañantes representan los años 2002 a 2005.

Figure 3. Seasonal variation in the diversity of bird species from 2002 to 2005 at the Carriel Sur Airport of Talcahuano, southern Chile. $\mathrm{V}=$ summer, $\mathrm{O}=$ autumn, $\mathrm{I}=$ winter, $\mathrm{P}=$ spring; the accompanying numbers represent the years 2002 to 2005 . más abundantes $(24 \%, 19 \%$ y $8 \%$, respectivamente; González-Acuña et al. 2006). Cabe señalar que el ABOC está emplazado en un área predominantemente agrícola lo cual explica la mayor abundancia de especies asociadas a pastizales y campos abiertos. La gaviota dominicana no fue registrada en el ABOC ya que esta especie se asocia fuertemente a ambientes marinos costeros. De hecho, esta especie fue la más abundante en el ACIC de Punta Arenas (Olivares 1995), el cual esta localizado relativamente cerca de la línea costera.

Con el propósito de realizar un análisis estacional, se seleccionaron las ocho especies más abundantes (las que tienen el IP más alto). Todas estas especies mostraron variaciones temporales evidentes (Fig. 5). Dos de las cuatro especies de paseriformes incluidas, el chirigüe y la loica, tendieron a incrementar considerablemente su abundancia hacia los meses de otoño y a disminuir hacia los meses de verano. En el último año, sin embargo, el chirigüe mostró una abundancia inusualmente alta durante la primavera. Aunque la gaviota dominicana no tuvo un patrón estacional cíclico exhibió dos fuertes irrupciones a lo largo del periodo de estudio. En general, el queltehue fue más abundante en otoño-invierno, pero durante el verano de 2004 su abundancia fue similar a la de los meses invernales. El yeco también tendió a ser más abundante hacia los meses de otoño e invierno exhibiendo un alza numérica considerable durante el otoño de 2002. La golondrina chilena tendió a ser más abundante durante los meses de primavera, aunque exhibiendo un fuerte incremento durante el verano de 2003. El jilguero mostró un claro patrón estacional en su abundancia tendiendo a ser mucho más abundante durante los meses de invierno. La abundancia de tiuque fue relativamente estable a lo largo del tiempo exhibiendo un alza leve durante el verano de 2002 e invierno de 2003. Estos resultados revelan que varias de las especies más abundantes en el área de influencia del ACST están sujetas a ciclos estacionales de abundancia, y de ahí sus variaciones numéricas pueden ser predecibles, mientras otras especies exhiben irrupciones numéricas acíclicas y, de ahí, sus variaciones numéricas no podrían ser predichas.

En general, las aves paserinas aumentan su abundancia en los meses de otoño como consecuencia del reclutamiento de individuos juveniles que tienden a disminuir su abundancia hacia fines del invierno e inicio de la primavera debido a la mortalidad por causas climáticas y/o depredación (e.g., Winter 2004, Davis 2005, Macías-Duarte et al. 2009). En el caso particular de chirigües y jilgueros, aumentan en áreas con pastizales abandonados, campos de sembradío, praderas ganaderas y matorrales (Housse 1945, Goodall et al. 1946, Couve \& Vidal 2003), lugares que ofrecen una alta disponibilidad de semillas comestibles en los meses fríos para el chirigüe y el jilguero, dos especies esencialmente 
granívoras (Estades \& Temple 1999). Además, las praderas ganaderas y campos de cultivo usualmente contienen una alta densidad de larvas de insectos y lombrices terrestres constituyendo importantes hábitats durante la época invernal de forrajeo de queltehues, loicas y tiuques. Junto a esto, en la temporada de lluvias muchos individuos de gaviotas, queltehues y tiuques se congregan en el terreno empastado alrededor de las pistas de aterrizaje para consumir las lombrices que emergen a la superficie por el exceso de precipitación (Olivares 1995). Las praderas empastadas con vegetación corta y escasa perturbación humana favorecen también el anidamiento de queltehues dentro de los límites del ACST. La disminución estival en el número de chirigües, loicas, jilgueros y queltehues puede deberse en parte a la acción de depredadores locales. También, podría haber una subestimación producto de que en esta época no forman bandadas y restringen sus movimientos a sus territorios de nidificación. Todas estas especies constituyen presas frecuentes de aves rapaces que cazan en áreas abiertas tales como el nuco, vari, peuco, cernícalo y halcón perdiguero (Martínez et al. 1998, Figueroa \& Corales 1999, 2004,
2005, Figueroa \& González-Acuña 2006).

El incremento explosivo de gaviotas dominicanas en el área de influencia del ACST durante el invierno de 2002 y verano de 2004 no tiene una explicación clara. Posiblemente durante esas estaciones las gaviotas usaron con mayor intensidad el espacio aéreo del aeropuerto como zona de tránsito hacia el vertedero Cosmito ubicado a $400 \mathrm{~m}$ en dirección noreste. Los vertederos de basura, particularmente aquellos que reciben desechos orgánicos comestibles, pueden constituir importantes fuentes de alimento para las gaviotas (Caccamise et al. 1996). Cabe señalar que después de clausurado el vertedero Cosmito (septiembre de 2004) no se han vuelto a observar grandes incrementos en el numero de gaviotas. Las variaciones estacionales en el número de queltehues en el ACST es consistente con lo observado por González-Acuña et al. (2006) en el ABOC de Chillán en que encuentran mayor abundancia de la especie en la época invernal, hecho que podría deberse a migraciones locales promovidas por la búsqueda de alimento y nuevos sitios de anidamiento (Godínez 2000).

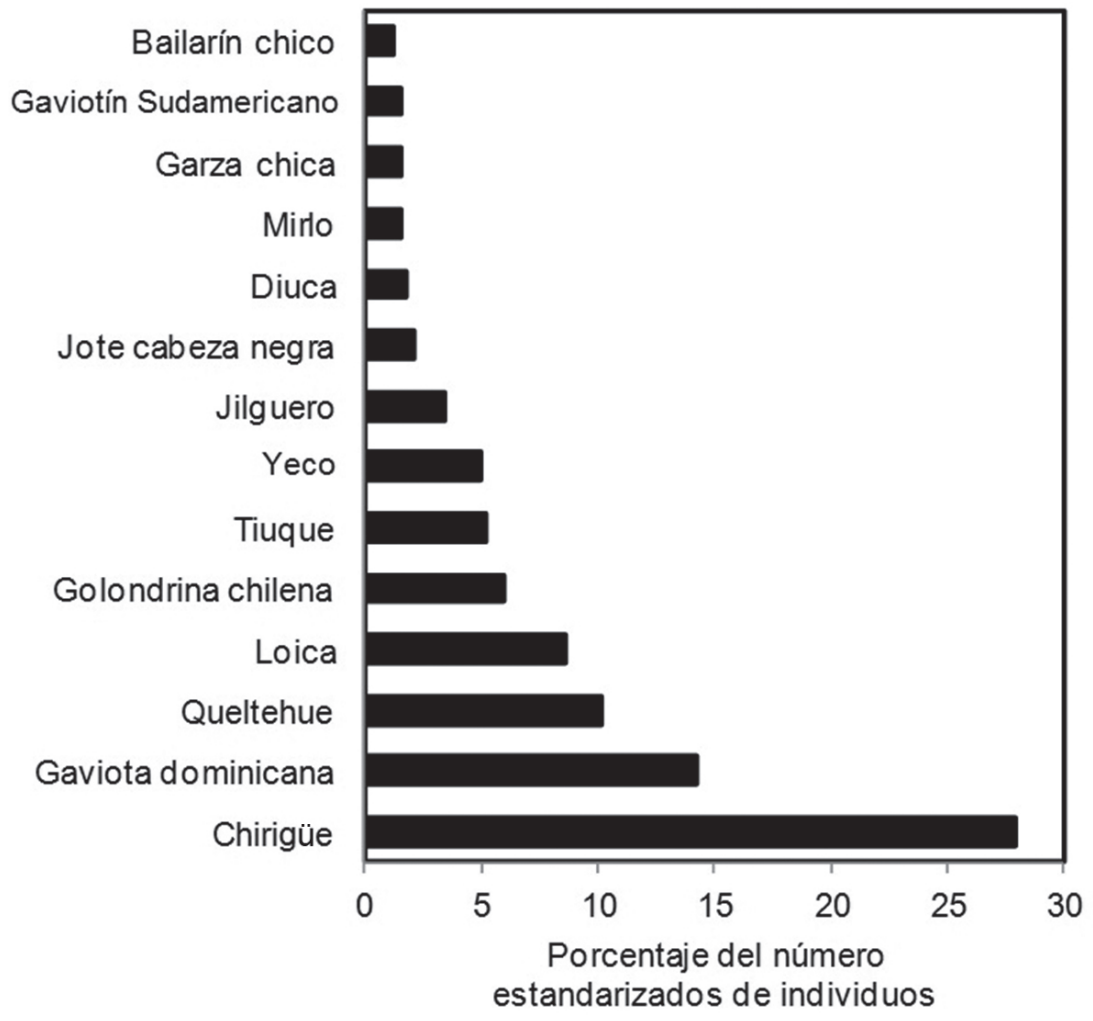

Figura 4. Rango de abundancia relativa de las especies más registradas entre los años 2002 y 2003 en el Aeropuerto Carriel Sur de Talcahuano, sur de Chile. El porcentaje de corte fue 1\%; aquellas especies con abundancia $<1 \%$ son indicadas en el Anexo 1.

Figure 4. Range of relative abundance of most recorded species during the years 2002 and 2003 at the Carriel Sur Airport of Talcahuano, southern Chile. The cutting percentage was $1 \%$; those species with less than $<1 \%$ are indicated in Appendix 1 . 

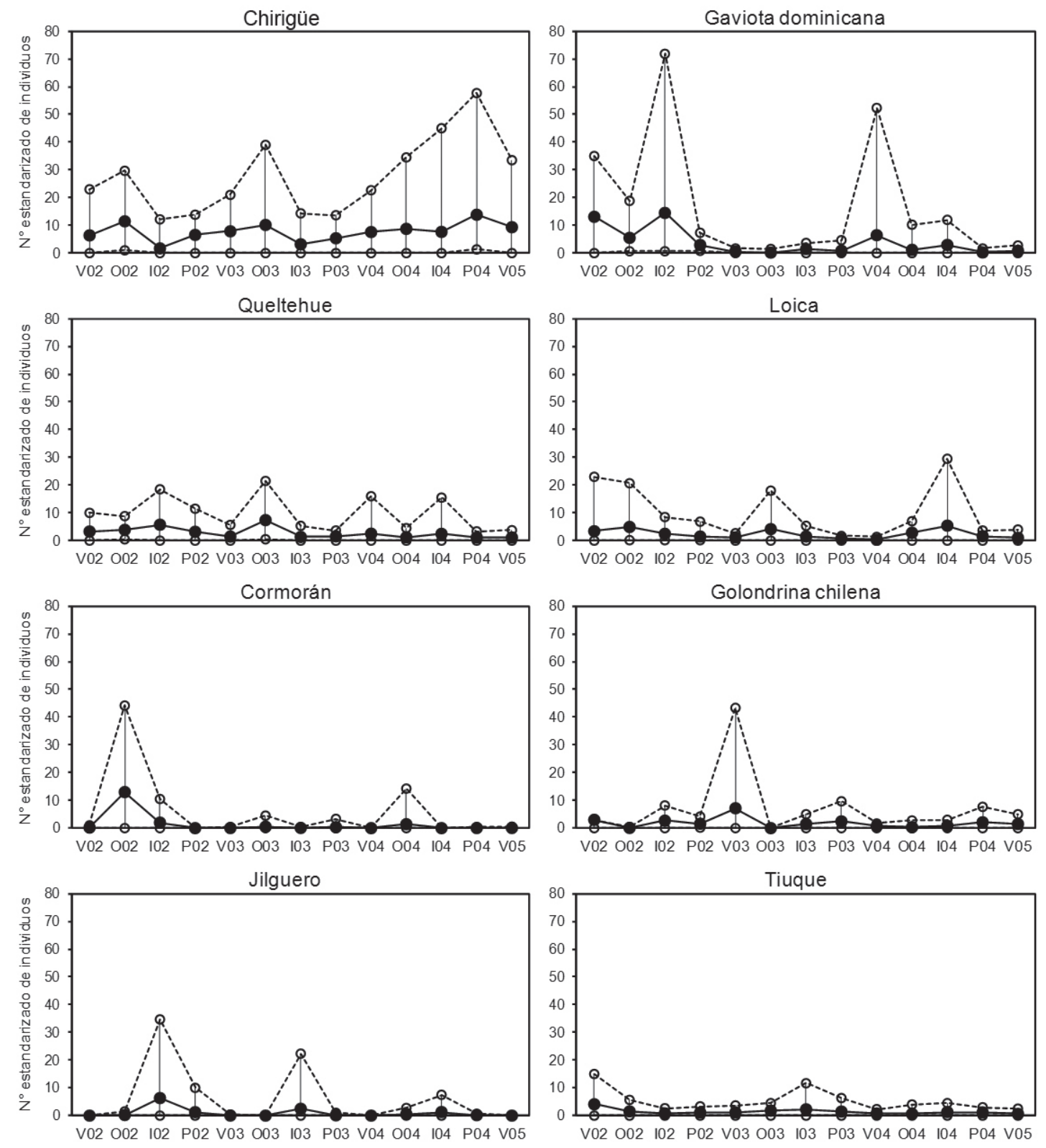

Figura 5. Variación temporal en la abundancia relativa de las ocho especies de aves más registradas entre 2002-2005 en el Aeropuerto Carriel Sur de Talcahuano, sur de Chile. La abundancia se expresa como el número estandarizados de individuos por estación (i.e., número de individuos dividido por el número de conteos). Los círculos negros representan valores medios y los círculos blancos representan valores máximos y mínimos. $\mathrm{V}$ = verano, $\mathrm{O}=$ otoño, $\mathrm{I}=$ invierno, $\mathrm{P}=$ primavera; los números acompañantes representan los años 2002 a 2005.

FiguRE 5. Seasonal variation in the relative abundance of the eight species of birds most recorded during 2002-2005 at the Carriel Sur Airport of Talcahuano, southern Chile. Abundance is expressed as the standardized number of individuals per season (i.e., number of individuals divided by the number of counts). The black circles represent mean values and the white circles represent maximum and minimum values. $\mathrm{V}=$ summer, $\mathrm{O}=$ autumn, $\mathrm{I}=$ winter, $\mathrm{P}=$ spring; the accompanying numbers represent the years 2002 to 2005. 

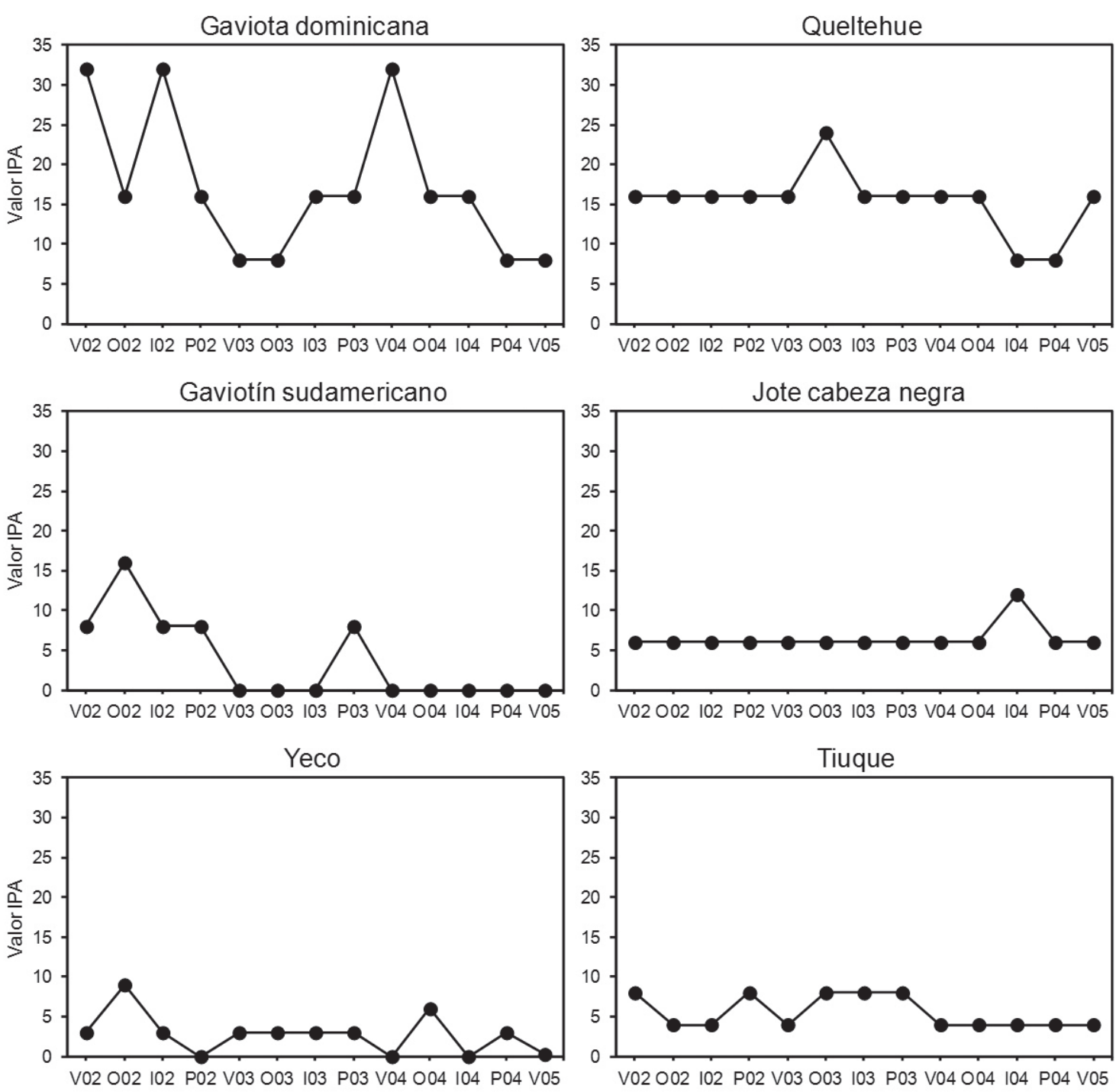

Figura 6. Variación temporal del índice de peligro aviario (IPA) de las seis especies de aves que alcanzaron el mayor IPA global en el Aeropuerto Carriel Sur de Talcahuano, sur de Chile. $\mathrm{V}=$ verano, $\mathrm{O}=$ otoño, $\mathrm{I}=$ invierno, $\mathrm{P}=$ primavera; los números acompañantes representan los años 2002 a 2005.

Figure 6. Seasonal variation of the index of avian danger (IPA) for six species of birds that reached the greatest overall IPA at the Carriel Sur Airport of Talcahuano, southern Chile. $\mathrm{V}=$ summer, $\mathrm{O}=$ autumn, $\mathrm{I}=$ winter, $\mathrm{P}=$ spring; the accompanying numbers represent the years 2002 to 2005.

ÍNDICE DE PELIGRO AVIARIO

Las aves que alcanzaron el mayor IPA fueron la gaviota dominicana, el queltehue, gaviotín sudamericano, jote de cabeza negra, yeco y tiuque (Tabla 1). Coincidentemente, varias de estas especies están entre aquellas que representan un mayor riesgo de colisión con aeronaves a escala nacional e internacional. La gaviota dominicana es considerada mundialmente como un ave de alto riesgo para las maniobras aéreas debido a su comportamiento gregario, tamaño relativamente grande, vuelos elevados y desplazamientos extensos (Dolbeer et al. 1993, Olivares 1995, Martelli 2000, Cleary \& Dolbeer 2005, Dolbeer 2006). El queltehue también ha sido identificado en algunos aeropuertos del país como una especie peligrosa para las maniobras de vuelo (Martelli 2000, Muñoz 2004, González-Acuña et al. 2006). Aunque el queltehue fue mucho menos numeroso que la gaviota dominicana, su tamaño corporal y su historial de colisiones hicieron que su IPA fuera alto. Aunque no tan abundante, el gaviotín sudamericano es una especie de tamaño relativamente grande (longitud corporal $=39-41$ 
cm; Goodall et al. 1951) que se desplaza en grande grupos dentro del espacio aéreo del ACST lo cual lo hace riesgoso para la aeronavegación. A diferencia de lo documentado por Martelli (2000), en este estudio el jote de cabeza negra fue categorizado como una especie riesgosa para las operaciones aéreas. Esta discrepancia se debería posiblemente a que su abundancia fue mayor durante nuestro estudio. El jote de cabeza negra posee al menos otras tres características que lo harían riesgoso para el tráfico aéreo en ACST: (i) es una de las especies de mayor tamaño en el área (longitud corporal = 61-68 cm; Goodall et al. 1951), (ii) es fuertemente gregario, y (iii) posee un vuelo lento y planeado el cual puede alcanzar grandes alturas mediante el uso corrientes térmicas (Figueroa et al. 2001; Couve \& Vidal 2003; Martínez \& González 2005).

El IPA de cada especie varió temporalmente según los cambios temporales de su abundancia. Sin embargo, excepto en el caso de la gaviota dominicana, las curvas de variación del IPA no siguieron estrechamente aquellas de la abundancia. Esto se debe a que estas últimas fueron ingresadas como valores categóricos en el cálculo los IPA y/o a que lo cambios temporales de abundancia no fueron tan marcados. Más allá de esto último, lo relevante es tener en cuenta que las poblaciones de aves en el área de influencia de los aeropuertos no son estáticas, por lo tanto tampoco la peligrosidad aviar. Esto implica la necesidad de evaluaciones de largo plazo de los cambios en el tamaño poblacional de especies riesgosas para una mejor aproximación del riesgo de colisiones aves-aeronaves (Bell 1999).

¿SE puede mitigar el RiESGo de Colisiones aVeS-AeronaVes EN EL ACST?

Al mismo tiempo que los aeropuertos ofrecen una diversidad de hábitats naturales bien protegidos (Godin 1994, Matijaca 2000), también pueden operar como trampas ecológicas para las aves. Un gran número de distintas especies de aves mueren por efecto de las colisiones con aeronaves en las distintas fases de las maniobras aéreas (Sheehy et al. 2005, Dolbeer 2006). Además, los protocolos de seguridad de los aeropuertos promueven la reducción poblacional de aves mediante distintas estrategias de manejo ambiental (Blackwell et al. 2009). De esta manera, compatibilizar la conservación de aves con la seguridad para la aviación en los aeropuertos constituye un desafío (Mackinnon 2002).

TABLA 1. Índice de Peligro Aviario (IPA) calculado para las especies de aves registradas entre los años 2002-2005 en el Aeropuerto Carriel Sur de Talcahuano, sur de Chile. El valor del IPA se obtuvo multiplicando las categorías de peso, abundancia y participación en colisiones.

TABLE 1. Index of Avian Danger (IPA) calculated for the bird species registered during the years 2002-2005 at the Carriel Sur Airport of Talcahuano, southern Chile. The value of the IPA was obtained by multiplying the categories of weight, abundance and participation in collisions.

\begin{tabular}{|c|c|c|c|c|}
\hline ESPECIES & Categoría del Peso & $\begin{array}{l}\text { CATEGORÍA DE } \\
\text { ABUNDANCIA }\end{array}$ & $\begin{array}{l}\text { CATEGORÍA DE } \\
\text { PARTICIPACIÓN EN } \\
\text { COLISIONES }\end{array}$ & IPA \\
\hline Gaviota dominicana & 2 & 4 & 4 & 32 \\
\hline Queltehue & 2 & 3 & 4 & 24 \\
\hline Gaviotín sudamericano & 2 & 2 & 4 & 16 \\
\hline Jote de cabeza negra & 3 & 2 & 2 & 12 \\
\hline Yeco & 3 & 3 & 1 & 9 \\
\hline Tiuque & 2 & 2 & 2 & 8 \\
\hline Pequén & 2 & 1 & 3 & 6 \\
\hline Chirigüe & 1 & 6 & 1 & 6 \\
\hline Garza chica & 2 & 2 & 1 & 4 \\
\hline Cisne cuello negro & 3 & 1 & 1 & 3 \\
\hline Aguilucho común & 3 & 1 & 1 & 3 \\
\hline Cisne coscoroba & 3 & 1 & 1 & 3 \\
\hline Garza cuca & 3 & 1 & 1 & 3 \\
\hline Golondrina chilena & 1 & 3 & 1 & 3 \\
\hline Halcón peregrino & 3 & 1 & 1 & 3 \\
\hline Jote cabeza colorada & 3 & 1 & 1 & 3 \\
\hline Loica & 1 & 3 & 1 & 3 \\
\hline Peuco & 3 & 1 & 1 & 3 \\
\hline Bailarín & 2 & 1 & 1 & 2 \\
\hline Becacina & 2 & 1 & 1 & 2 \\
\hline Diuca & 1 & 2 & 1 & 2 \\
\hline Garza boyera & 2 & 1 & 1 & 2 \\
\hline
\end{tabular}




\begin{tabular}{|c|c|c|c|c|}
\hline ESPECIES & Categoría del Peso & $\begin{array}{l}\text { CATEGoRÍA DE } \\
\text { ABUNDANCIA }\end{array}$ & $\begin{array}{c}\text { CATEGORÍA DE } \\
\text { PARTICIPACIÓN EN } \\
\text { COLISIONES }\end{array}$ & IPA \\
\hline Garza grande & 2 & 1 & 1 & 2 \\
\hline Jilguero & 1 & 2 & 1 & 2 \\
\hline Mirlo & 1 & 2 & 1 & 2 \\
\hline Paloma & 2 & 1 & 1 & 2 \\
\hline Pato jergón grande & 2 & 1 & 1 & 2 \\
\hline Perdiz & 2 & 1 & 1 & 2 \\
\hline Tagua & 2 & 1 & 1 & 2 \\
\hline Vari & 2 & 1 & 1 & 2 \\
\hline Cachaña & 2 & 1 & 1 & 2 \\
\hline Nuco & 2 & 1 & 1 & 2 \\
\hline Pato Colorado & 2 & 1 & 1 & 2 \\
\hline Pato gargantillo & 2 & 1 & 1 & 2 \\
\hline Pato jergón chico & 2 & 1 & 1 & 2 \\
\hline Pato real & 2 & 1 & 1 & 2 \\
\hline Perrito & 2 & 1 & 1 & 2 \\
\hline Pilpilén & 2 & 1 & 1 & 2 \\
\hline Pidén & 2 & 1 & 1 & 2 \\
\hline Zarapito & 2 & 1 & 1 & 2 \\
\hline Bailarín chico & 1 & 1 & 1 & 1 \\
\hline Cernícalo & 1 & 1 & 1 & 1 \\
\hline Chercán & 1 & 1 & 1 & 1 \\
\hline Chincol & 1 & 1 & 1 & 1 \\
\hline Codorniz & 2 & 1 & 1 & 1 \\
\hline Dormilona tontita & 1 & 1 & 1 & 1 \\
\hline Golondrina dorso negro & 1 & 1 & 1 & 1 \\
\hline Gorrión & 1 & 1 & 1 & 1 \\
\hline Picaflor & 1 & 1 & 1 & 1 \\
\hline Run-Run & 1 & 1 & 1 & 1 \\
\hline Tagüita & 1 & 1 & 1 & 1 \\
\hline Tenca & 1 & 1 & 1 & 1 \\
\hline Tordo & 1 & 1 & 1 & 1 \\
\hline Tórtola & 1 & 1 & 1 & 1 \\
\hline Trile & 1 & 1 & 1 & 1 \\
\hline Zorzal & 1 & 1 & 1 & 1 \\
\hline Cachudito & 1 & 1 & 1 & 1 \\
\hline Chercán de las vegas & 1 & 1 & 1 & 1 \\
\hline Churrete & 1 & 1 & 1 & 1 \\
\hline Churrete acanelado & 1 & 1 & 1 & 1 \\
\hline Colegial & 1 & 1 & 1 & 1 \\
\hline Diucón & 1 & 1 & 1 & 1 \\
\hline Fío-fío & 1 & 1 & 1 & 1 \\
\hline Minero & 1 & 1 & 1 & 1 \\
\hline Picurio & 1 & 1 & 1 & 1 \\
\hline Pimpollo & 1 & 1 & 1 & 1 \\
\hline Platero & 1 & 1 & 1 & 1 \\
\hline Rara & 1 & 1 & 1 & 1 \\
\hline Tijeral & 1 & 1 & 1 & 1 \\
\hline Tortolita Cuyana & 1 & 1 & 1 & 1 \\
\hline Trabajador & 1 & 1 & 1 & 1 \\
\hline Yal & 1 & 1 & 1 & 1 \\
\hline
\end{tabular}


En el caso particular del ACST, la alta diversidad de hábitat naturales tanto acuáticos como terrestres favorece fuertemente la existencia de una avifauna rica y abundante. Desde el punto de vista aeronáutico, esto es un problema de seguridad para la aeronavegación. Dada las condiciones ecológicas en los alrededores del ACST podría ser difícil lograr medidas para reducir las poblaciones de todas las especies de aves potencialmente riesgosas. Por ejemplo, es difícil controlar la oferta de alimento generada por la industria pesquera que favorecería particularmente a la gaviota dominicana. Sin embargo, la eliminación de basurales o vertederos cercanos a los aeropuertos si puede resultar en medidas de mitigación relativamente efectivas. El hecho que la abundancia de gaviotas dominicanas en el ACST haya disminuido aparentemente después de la clausura de un basural muy cercano sugiere que medidas de carácter administrativo son relevantes para la seguridad en los aeropuertos (Bitebekezi 2007). Otra medida relativamente efectiva para reducir el número de gaviotas es el removimiento o destrucción de huevos (Blokpoel \& Tessier 1987), pero esta medida puede generar rechazo por parte de la ciudadanía. Además, esta medida debe considerar un manejo de hábitat, con el fin de que las aves no sigan ocupando los mismos sitios. Los presencia de animales muertos y acumulaciones de desechos comestibles en los predios privados cercanos a los aeropuertos que atraen a jotes y tiuques también pueden ser regulados por las autoridades sanitarias. De esta manera, el manejo de desechos orgánicos en las cercanías de los aeropuertos puede ser facilitado mediante una trabajo coordinado entre las autoridades aeronáuticas, servicios públicos y la comunidad local (Bitebekezi 2007, Blackwell et al. 2009).

Un caso desafiante es el del queltehue. Los prados que bordean las pistas de aterrizaje y terrenos empastados son sus hábitats de forrajeo y anidamiento preferidos (Rottmann 1995, Figueroa et al. 2001b, Cabello 2004). Además, los queltehues aprovechan las pistas de aterrizaje como fuentes de calor y los techos de las edificaciones como punto de vigilancia. Todos estos factores aseguran un número alto y estable dentro de los límites del ACST. La reducción de la población de queltehues podría lograrse mediante la disminución de la disponibilidad de presas y sitios de anidamiento. Sin embargo, la cantidad de presas sólo podría ser reducida efectivamente mediante insecticidas, una medida ambientalmente prohibitiva ya que puede afectar a muchas otras especies insectívoras no riesgosas. Algunas medidas viables son el uso de perros Border Collie para ahuyentar y desincentivar la presencia de queltehues, y el uso de halcones entrenados para aumentar la mortalidad por depredación (Solman 1973, Ryan 1999, Patterson 2000). Repelentes químicos con efectos irritantes (e.g., metil-antranilato) también han sido usados dentro de los aeropuertos para dispersar aves en general y avefrías (Vanellus spp.) en particular (Milsom \&
Horton 1995; Engeman et al. 2002, Vogt 2000), sin embargo esta metodología no ha sido probada en Chile y habría que situarla en el contexto legal. Una alternativa menos invasiva es el uso de prados artificiales o de especies de pastos que minimicen la presencia de invertebrados (Linnel et al. 2009).

Otras medidas de mitigación incluyen el drenaje de humedales, manejo de pastizales, reducción de presas, removimiento de nidos y manejo de elementos estructurales (Dolbeer et al. 1989, Caccamise et al. 1996, Harris \& Davis 1998, Barras \& Seamans 2002, Sodhi 2002, Cleary \& Dolbeer 2005, Bitebekezi 2007). El drenaje de cuerpos de agua, tanto permanentes como temporales, podría ser importante para reducir la presencia de gaviotas (Dolbeer et al. 1989). La reducción de la altura o densidad de pastizales puede reducir la presencia de algunas especies paserinas (Barras \& Seamans 2002), pero aumentar el número de queltehues. En cambio, los pastizales altos pueden disminuir la presencia de queltehues, pero puede favorecer la disponibilidad de roedores y así atraer a las aves rapaces (Barras \& Seamans 2002, Witmer \& Fantinato 2003). El removimiento de nidos ha sido aplicado en el Aeropuerto Internacional de Entebbe, Uganda, aparentemente con éxito (Bitebekezi 2007). Existen pocos estudios acerca del manejo de los elementos estructurales (e.g., postes, vigas, torres) para minimizar la presencia de aves en los aeropuertos (Sodhi 2002). La reducción de la cantidad de cavidades estructurales podría minimizar la presencia de especies que utilizan oquedades para refugiarse y anidar (e.g., golondrinas, gorriones, chercanes, cernícalos).

Aún cuando todas estas medidas son importantes para reducir la peligrosidad aviar, podrían tener un valor limitado para reducir las colisiones causadas por especies determinadas o en condiciones ambientales particulares (Dolbeer et al. 1989, Sodhi 2002). Sin duda, aún queda mucho por avanzar en cuanto a medidas de mitigación en el ACST y esto debe implicar el desarrollo de un manejo integrado y adaptativo que incluya la asesoría permanente de un panel de expertos (Vasilis et al. 2005). Se sugiere en futuros estudios, y con el fin de tener mayor precisión de las horas de mayor flujo de las aves, realizar un monitoreo que abarque todas las horas de actividad en el ACST, de esta forma se entenderían además las rutas y conductas de las aves de mayor riesgo. Además, debe existir un trabajo mancomunado entre las autoridades aeronáuticas y los distintos componentes de la comunidad civil afectada por la actividad aeroportuaria (Bitebekesi 2007). Debido a que la ciudadanía siente cada vez más aprecio por las aves silvestres y entiende su rol en los ecosistemas, un aspecto relevante es educar a la ciudadanía acerca de las medidas de mitigación en los aeropuertos explicando porque tales medidas son implementadas y porque algunas aves podrían ser peligrosas para las maniobras aéreas. 


\section{AGRADECIMIENTOS}

A Mauricio Muñoz y personal del Aeropuerto Carriel Sur por las facilidades otorgadas durante este estudio. Parte de las observaciones se realizaron durante el estudio "las gaviotas como vectores de enfermedades zoonoticas en Talcahuano", financiado por el Proyecto Fondecyt 1070664.

\section{BIBLIOGRAFÍA}

Allan, J.R. 2000. The costs of bird strikes and bird strike prevention. Human Conflicts with Wildlife: Economic Considerations. USDA National Wildlife Research Center Symposia, Paper 18.

Araya, B., Bernal, M., Schlatter, R. \& Sallaberry, M. 1995. Lista patrón de las aves chilenas. Tercera edición. Santiago, Chile. 35 pp.

BAKer, J.A. \& BRooks, R.J. 1981. Raptor and vole populations at an airport. Journal of Wildlife Management 45:390-396.

Barras, S.C. \& SEAmans, T.W. 2002. Habitat management approaches for reducing wildlife use of airfields. In: Proceedings of the $20^{\text {th }}$ Vertebrate Pest Conference (Eds. Timm, R.M \& Schmidt, R.H.), pp. 309-315. Davis, California.

BELL, J.C. 1999. The effects of changes in the Northern Lapwing population on the bird strike hazard in the UK. Bird Strike Committee-USA/Canada, First Joint Annual Meeting, Vancouver, BC. Paper 9.

Bibby, C., Burgess, N. \& Hill, D.A. 2000. Bird census techniques. Academic Press Inc, 2da edition, London, U.K. 302 pp.

BiteBeKeZI, G.K. 2007. The role of community participation in the control of bird hazards at Entebbe International Airport, Uganda. Ostrich 78:131-133.

Blackwell, B.F, DeVault, T.L., Fernández-Juricic, E. \& Dolbeer, R.A. 2009. Wildlife collisions with aircraft: a missing component of land-use planning for airports. Landscape and Urban Planning 93:1-9.

Blokpoel, H. \& Tessier, G.D. 1987. Control of ring-billed gull colonies at urban and industrial sites in southern ontario, canada. Third Eastern Wildlife Damage Control Conference. Pp. 8-17.

Cabello, J. 2004. Aspectos reproductivos de Vanellus chilensis (Aves, Molina, 1782) en Ñuble, Chile. Memoria de título. Universidad de Concepción, Chillán, Chile.

Caccamise, D.F., Reed, L.M. \& Romanowsky, J. 1996. Bird strike hazards and waste management facilities in urban landscapes. Proceedings of the International Bird Strike Committee 23:247-266.

Cleary, E. \& Dolbeer, R.A. 2005. Wildlife Hazard Management at Airports. 2nd Edition. Federal Aviation Administration, Office of Airport Safety and Standards, Airport Safety and Compliance Branch, Washington, DC, USA. 389 pp.

Couve, E. \& Vidal, C. 2003. Aves de Patagonia, Tierra del Fuego y Península Antártica: Islas Malvinas y Georgia del Sur. Fantástico Sur Birding Ltda. Punta Arenas, Chile. 656 pp.

DAVIS, S.K. 2005. Nest-site selection patterns and the influence of vegetation on nest survival of mixed-grass prairie passerines. Condor 107:605-616
Dekker, A. 2000. Poor long grass: low bird density ground cover for the runway environment. Proceedings of the International Bird Strike Conference 25:227-236.

Di Castri, F. \& HajeK, E. 1976. Bioclimatología de Chile. Universidad Católica de Chile. Santiago, Chile. 128 pp.

Dolbeer, R.A. 2006. Height distribution of birds recorded by collisions with civil aircraft. Journal of Wildlife Management 70:1345-1350.

Dolbeer, R.A., Belant, J.L. \& Silling, J.L. 1993. Shooting gulls reduces strikes with aircraft at John F. Kennedy international airport. Wildlife Society Bulletin 21:442-450.

Dolbeer, R.A., Chevalier, M., Woronecki, P.P. \& Butler, E.B. 1989. Laughing gulls at JFK Airport: safety hazard or wildlife resource? Fourth Eastern Wildlife Damage Control Conference. Pp.37-44.

Dolbeer, R.A., Wright, S.E. \& Cleary, E.C. 2000. Ranking the hazard level of wildlife species to aviation. Wildlife Society Bulletin 28:372-378.

Engeman, R.M., Peterla, J. \& Constantin, B. 2002. Methyl anthranilate aerosol for dispersing birds from the flight lines at Homestead Air Reserve Station. International Biodeterioration \& Biodegradation 49:175-178.

Estades, C. 1994. Impacto de la sustitución del bosque natural por plantaciones de Pinus radiata sobre una comunidad de aves en la octava región de Chile. Boletín Chileno de Ornitología 1:8-14

Estades, C.F. \& Temple, S.A. 1999. Deciduous-forest bird communities in a fragmented landscape dominated by exotic pine plantations. Ecological Applications 9:573-585.

Figueroa, R.A. \& Corales, E.S. 1999. Food habits of the Cinereous Harriers (Circus cinereus) in the Araucanía, southern Chile. Journal of Raptors Research 33:264-267.

Figueroa, R.A. \& Corales, E.S. 2004. Summer diet comparison of the American Kestrel (Falco sparverius) and Aplomado Falcon (Falco femoralis) in an agricultural area of Araucanía, southern Chile. Hornero 19:53-60.

Figueroa, R.A. \& Corales, E.S. 2005. Seasonal diet of the Aplomado Falcon (Falco femoralis) in an agricultural area of Araucanía, southern Chile. Journal of Raptor Research 39:55-60.

Figueroa, R.A. \& GonzÁlez-AcuÑA, D. 2006. Prey of the Harris' Hawk (Parabuteo unicinctus) in a suburban area of southern Chile. Journal of Raptor Research 40:164-168

Figueroa, R.A. \& QuintanA, V. 2001. Comunidad invernal de aves en un paisaje agroforestal del centro-sur de Chile. Boletín Chileno de Ornitología 8:31-35.

Figueroa, R.A, Corales, S., Cerda, J. \& Saldivia, H. 2001a. Roedores, rapaces y carnívoros de Aysén. Segunda Edición. Servicio Agrícola y Ganadero, Aysén, Chile. 192 pp.

Figueroa, R.A., Cerda, J. \& Tala, C. 2001b. Guía de aves dulceacuícolas de Aysén. Servicio Agrícola y Ganadero de Aysén, Ministerio de Agricultura, Chile. 184 pp

Fowler, J. \& CoHEN, L. 1995. Statistics for ornithologists. British Trust for Ornithology, U.K. 176 pp.

Garland, H., del Peral, A. \& Bird, D.M. 2009. High frequency of American Kestrel strikes at the Montreal-Trudeau International Airport: a case study. Journal of Raptor Research 43:382-383.

Godin, A.J. 1994. Prevention and control of wildlife damage. University of Nebraska. Linconls, USA.

Godínez, E. 2000. Vanellus chilensis en Panamá. Boletín Chileno 
de Ornitología 7:31.

GonzÁlez-Acuña, D., Valenzuela-Dellarossa, G., Barrientos, C., Ardiles, K., Godoy, C. \& Figueroa, R.A. 2006. Aves del Aeródromo Bernardo O`Higgins de Chillán, Región del Bío-Bío, Chile. Boletín Chileno de Ornitología 12:15-25.

González-Acuña, D, Briones, E., Valenzuela-Dellarossa, G., Corales, E.S. \& Figueroa, R.A. 2009. Seasonal variation in the diet of the White-tailed Kite (Elanus leucurus) in a suburban area of southern Chile. Journal of Raptor Research 43:134-141.

González-Gajardo, A., Victoriano, P. \& Schlatter, P. 2009. Waterbird assemblages and habitat characteristics in wetlands: influence of temporal variability on specieshabitat relationships. Waterbirds 32:225-233.

Goodall, J.D, Johnson, A.W. \& Philippi, R.A. 1946. Las aves de Chile. Vol.I. Establecimientos Gráficos Platt S.A., Buenos Aires, Argentina.

Goodall, J.D, Johnson, A.W. \& Philippi, R.A. 1951. Las aves de Chile. Vol.II. Establecimientos Gráficos Platt S.A., Buenos Aires, Argentina.

Guthery, F.S., Lusk, J.J. \& Peterson, M.J. 2001. The fall of the null hypothesis: liabilities and opportunities. Journal of Wildlife Management 65:379-384.

HARris, R.E. \& DAvIS, R.A. 1998. Evaluation of the efficacy of products and techniques for airport bird control. Aerodrome Safety Branch Transport Canada, Ontario. 107 pp.

Housse, R.E. 1945. Las aves de Chile en su clasificación moderna. $\mathrm{Su}$ vida y costumbres. Ediciones Universidad de Chile. $390 \mathrm{pp}$.

Linnel, M.A., Conover, M.R. \& OHAshi, T.J. 2009. Using wedelia as ground cover on tropical airports to reduce bird activity. Human-Wildlife Conflict 3:226-236.

Macías-Duarte, A., Montoya, A.B., Méndez-González, C.E., Rodríguez-Salazar, J.R., Hunt, W.G. \& Krannitz, P.G. 2009. Factors influencing habitat use by migratory grassland birds in the state of Chihuahua, Mexico. Auk 126:896-905.

MacKinnon, B. 2002. Wildlife Control Procedures Manual. Transport Canada, Civil Aviaton. Ottawa, Canadá. 270 pp.

MagalhãEs, L.C. 2000. Brazilian avian hazard control programeducational initiatives. Proceedings of the International Bird Strike Committee 25:383-400.

Martelli, N. 2000. Enfoque territorial del problema del peligro aviario en aeropuerto Carriel Sur Concepción. Memoria de Título. Universidad de Chile. Santiago, Chile.

Martínez. D.R., Figueroa, R.A., Ocampo, C.L. \& JaKsic, F.M. 1998. Food habits and hunting ranges of Short-eared Owls (Asio flammeus) in human-altered environments of southern Chile. Journal of Raptor Research 32:111-115.

Martínez, D. \& GonzÁlez, G. 2005. Las aves de Chile, Nueva guía de campo. Ediciones del naturalista. Santiago, Chile. 620 pp.

MatijacA, A. 2000. Air traffic safety concerning threat of collision of birds and aircraft, with regard to the situation in the republic of Croatia. Proceedings of the International Bird Strike Committee 25:195-204.

Milsom, T.P. \& Horton, N. 1995. Birdstrike - an assessment of the hazard on UK civil aerodromes 1976-1990. Central Sciencie Laboratory, Slough.

MuÑoz, A. 2004. Metodología para las especificaciones técnicas de los estudios científicos orientados a la mitigación y evaluación de riesgos por fauna silvestre en el Aeropuerto Arturo Merino Benítez. Universidad Católica de Temuco. Escuela de Ciencias Ambientales. Temuco, Chile.

Olivares, H.C. 1995. Peligro aviario en el Aeropuerto Carlos Ibañez del Campo: Proyecto de aplicación presentado en conformidad a las medidas que deben ser adoptadas sobre prevención del peligro de las aves en aeropuertos y aeródromos. Dirección General de Aeronáutica Civil. Punta Arenas, Chile.

Rau, J.R., Zuleta, C., Gantz, A., Saiz, F., Cortes, A., Yates, L., Spotorno, A.E. \& Couve, E. 1998. Biodiversidad de artrópodos y vertebrados terrestres del Norte Grande de Chile. Revista Chilena de Historia Natural 71:527-554.

Patterson, B. 2000. Wildlife control at Vancouver International Airport: Introducing border collies. International Bird Strike Committee IBSC25/WP-A6:1-7.

Remsen, J.V., Jr., Cadena, C.D., Jaramillo, A., Nores, M., Pacheco, J.F., Pérez-Emán, J., Robbins, M.B., Stiles, F.G., Stotz, D.F. \& Zimmer, K.J. 2013. A classification of the bird species of South America. American Ornithologists' Union. www.museum.lsu.edu/ Remsen/ SACCBaseline. html

Rottmann, J. 1995. Guía de identificación de aves de ambientes acuáticos. Unión de Ornitólogos de Chile. Santiago, Chile. $77 \mathrm{pp}$.

RYAN, L. 1999. Jet the border collie invites birds to leave airport. Airports Americas 7:10.

Sheehy, S., Kelly, T.C., Fennessy, G., O’Callaghan, M.J.A. \& Bolger, R. 2005. Birds strike syndrome: toward developing an index of bird injury. Proceedings of the International Bird Strike Committee 27:1-8.

Silva-Rodríguez, E.A., Ortega-Solís, G.R. \& Jiménez, J.E. 2008. Descripción del ensamble de aves en un agroecosistema del sur de Chile. Boletín Chileno de Ornitología 14:81-91.

SodHI, N.S. 2002. Competition in the air: birds versus aircraft. Auk 119:587-595.

Solman, V.E.F. 1973. Birds and aircraft. Biological Conservation 5:79-86.

Thorpe, J. 2003. Fatalities and destroyed civil aircraf due to bird strikes, 1912-202. Proceedings of the International Bird Strike Committee 26:1-28.

Vasilis, L., Jerrentrup, H., Dora. P. \& Anastasios, E. 2005. Assessment and integrated risk management of collissions aircrafts to birds at International Civil Aerodrome of Kavala (N.E. Greece). Proceedings of the International Bird Strike Committee 27:1-16.

Victoriano, P., GonzÁlez, A. \& Schlatter, R. 2006. Estado de conocimiento de las aves de aguas continentals de Chile. Gayana 70: 140-162.

Vogt, P.F. 2000. Control of nuisance birds in the airport arena by fogging with rejex-it TP-40/WS-40. Proceedings of the International Bird Strike Committee 25:297-304.

Winter, M, Johnson, D.H., Shaffer, J.A, \& Svedarsky, W.D. 2004. Nesting biology of three grassland passerines in the northern tallgrass prairie. Wilson Bulletin 116:211-223.

Witmer, G.W. \& Fantinato, J.W. 2003. Management of rodent population at airports. In: Proceeding of the $10^{\text {th }}$ Wildlife Damage Management Conference (Eds. Fagerstone, K.A. \& Witmer, G.W.), pp. 350-358. Hot Spring, AR. The Wildlife Damage Management Working Group of the Wildlife Society. Fort Collins, CO, USA. 
ANEXo 1. Lista completa de las especies de aves registradas en el aeropuerto Carriel Sur de Talcahuano, sur de Chile. Dieta: C = carnívoro $(\mathrm{a}=$ aves, $\mathrm{c}=$ crustáceos, $\mathrm{cr}=$ carroña, $\mathrm{i}=$ invertebrados, $\mathrm{m}=$ moluscos, $\mathrm{p}=$ peces, $\mathrm{r}=$ roedores $), \mathrm{F}=$ frugívoro, $\mathrm{G}=$ granívoro, $\mathrm{H}=$ herbívoro, $\mathrm{I}=$ insectívoro, $\mathrm{N}=$ nectarívoro, $\mathrm{O}=$ omnívoro. El orden de los acrónimos indica la preferencia trófica. Presencia estacional: $\mathrm{PV}$ = observado en primavera y verano, $\mathrm{OI}=$ observado en otoño e invierno, TA = observado durante todo el año.

ApPENDIX 1. Complete list of bird species recorded at the Carriel Sur Airport of Talcahuano, southern Chile. Diet: $\mathrm{C}=$ carnivorous $(\mathrm{a}=$ birds, $\mathrm{c}=$ crustaceans, $\mathrm{cr}=$ carrion, $\mathrm{i}=$ invertebrates, $\mathrm{m}=$ mollusks, $\mathrm{p}=$ fish, $\mathrm{r}=$ rodents), $\mathrm{F}=$ fruit-eating, $\mathrm{G}=$ seed-eating, $\mathrm{H}=$ herbivorous, $\mathrm{I}=$ insectivorous, $\mathrm{N}$ = nectarivorous, $\mathrm{O}=$ omnivorous. The order of the acronyms indicates the trophic preference. Seasonal presence: PV $=$ observed in spring and summer, $\mathrm{OI}=$ observed in autumn and winter, $\mathrm{TA}=$ observed throughout the year.

\begin{tabular}{|c|c|c|c|}
\hline NOMBRE CIENTÍFICO $^{\mathrm{a}}$ & NOMBRE COMÚN & DIETA $^{c}$ & Presencia estacional \\
\hline \multicolumn{4}{|l|}{ Orden Tinamiformes } \\
\hline Nothoprocta perdicaria & Perdiz chilena & $\mathrm{IH}$ & TA \\
\hline \multicolumn{4}{|l|}{ Orden Galliformes } \\
\hline Callipepla californica & Codorniz & & PV \\
\hline \multicolumn{4}{|l|}{ Orden Anseriformes } \\
\hline Anas bahamensis ${ }^{\mathrm{b}}$ & Pato gargantillo & $\mathrm{HI}$ & OI \\
\hline Anas cyanoptera ${ }^{\mathrm{b}}$ & Pato colorado & $\mathrm{O}$ & $\mathrm{PV}$ \\
\hline Anas flavirostris ${ }^{\mathrm{b}}$ & Pato jergón chico & $\mathrm{O}$ & OI \\
\hline Anas georgica & Pato jergón grande & $\mathrm{O}$ & TA \\
\hline Anas sibilatrix & Pato real & $\mathrm{H}$ & OI \\
\hline Cygnus melancoryphus & Cisne de cuello negro & $\mathrm{H}$ & OI \\
\hline Coscoroba coscoroba & Cisne coscoroba & HI & OI \\
\hline \multicolumn{4}{|l|}{ Orden Podicipediformes } \\
\hline Podylimbus podiceps & Picurio & $\mathrm{C}(\mathrm{i}, \mathrm{c}, \mathrm{p})$ & OI \\
\hline Rollandia rolland & Pimpollo & $\mathrm{C}(\mathrm{i}, \mathrm{c}, \mathrm{p})$ & $\mathrm{TA}$ \\
\hline \multicolumn{4}{|l|}{ Orden Suliformes } \\
\hline Phalacrocorax brasilianus & Yeco & $\mathrm{C}(\mathrm{p})$ & TA \\
\hline \multicolumn{4}{|l|}{ Orden Pelecaniformes } \\
\hline Bubulcus ibis & Garza boyera & C(i) & $\mathrm{TA}$ \\
\hline Egretta thula & Garza chica & $\mathrm{C}(\mathrm{p}, \mathrm{c}, \mathrm{i})$ & $\mathrm{TA}$ \\
\hline Ardea cocoi & Garza cuca & $\mathrm{C}(\mathrm{p}, \mathrm{c}, \mathrm{i})$ & OI \\
\hline Ardea alba & Garza grande & $\mathrm{C}(\mathrm{p}, \mathrm{c}, \mathrm{i})$ & TA \\
\hline \multicolumn{4}{|l|}{ Orden Cathartiformes } \\
\hline Cathartes aura & Jote de cabeza colorada & $\mathrm{C}(\mathrm{cr})$ & TA \\
\hline Coragyps atratus & Jote de cabeza negra & $\mathrm{C}(\mathrm{cr})$ & TA \\
\hline \multicolumn{4}{|l|}{ Orden Accipitriformes } \\
\hline Circus cinereus & Vari & $C(a, r)$ & $\mathrm{TA}$ \\
\hline Elanus leucurus & Bailarín & $\mathrm{C}(\mathrm{r})$ & TA \\
\hline Geranoaetus polyosoma & Aguilucho & $\mathrm{C}(\mathrm{r})$ & $\mathrm{TA}$ \\
\hline Parabuteo unicinctus & Peuco & $\mathrm{C}(\mathrm{a}, \mathrm{r})$ & TA \\
\hline \multicolumn{4}{|l|}{ Orden Falconiformes } \\
\hline Falco sparverius & Cernícalo & $\mathrm{C}(\mathrm{a}, \mathrm{r}, \mathrm{i})$ & TA \\
\hline Falco peregrinus $^{\mathrm{b}}$ & Halcón peregrino & $\mathrm{C}(\mathrm{a})$ & OI \\
\hline Milvago chimango & Tiuque & $\mathrm{C}(\mathrm{i})$ & $\mathrm{TA}$ \\
\hline \multicolumn{4}{|l|}{ Orden Gruiformes } \\
\hline Pardirallus sanguinolentus ${ }^{\mathrm{b}}$ & Pidén & I & TA \\
\hline Fulica armillata & Tagua & $\mathrm{H}$ & TA \\
\hline Gallinula melanops & Tagüita & $\mathrm{IH}$ & TA \\
\hline \multicolumn{4}{|l|}{ Orden Charadriformes } \\
\hline Haematopus palliatus $^{\mathrm{b}}$ & Pilpilén & $C(i, m)$ & OI \\
\hline Himantopus mexicanus $^{\mathrm{b}}$ & Perrito & I & OI \\
\hline Vanellus chilensis & Queltehue & I & TA \\
\hline Gallinago paraguaiae & Becacina & I & TA \\
\hline
\end{tabular}




\begin{tabular}{|c|c|c|c|}
\hline NOMBRE CIENTíFICO ${ }^{\mathrm{a}}$ & NOMBRE COMÚN & DIETA $^{c}$ & PRESENCIA ESTACIONAL \\
\hline Numenius phaeopus ${ }^{\mathrm{b}}$ & Zarapito & $C(\mathrm{c}, 1)$ & OI \\
\hline Chroicocephalus maculipennis ${ }^{\mathrm{b}}$ & Gaviota cáhuil & $\mathrm{C}(\mathrm{cr})$ & $\mathrm{OI}$ \\
\hline Larus dominicanus & Gaviota dominicana & $\mathrm{C}(\mathrm{cr})$ & TA \\
\hline Sterna hirundinacea & Gaviotín sudamericano & $\mathrm{C}(\mathrm{p}, \mathrm{i})$ & TA \\
\hline \multicolumn{4}{|l|}{ Orden Columbiformes } \\
\hline Columba livia & Paloma común & $\mathrm{O}$ & TA \\
\hline Zenaida auriculata & Tórtola & G & TA \\
\hline Columbina picui & Tortolita cuyana & G & TA \\
\hline \multicolumn{4}{|l|}{ Orden Psittaciformes } \\
\hline Enicognathus ferrugineus ${ }^{\mathrm{b}}$ & Cachaña & GF & PV \\
\hline \multicolumn{4}{|l|}{ Orden Strigiformes } \\
\hline Asio flammeus & Nuco & $\mathrm{C}(\mathrm{r}, \mathrm{a})$ & TA \\
\hline Athene cunicularia & Pequén & $\mathrm{C}(\mathrm{r}, \mathrm{i})$ & TA \\
\hline \multicolumn{4}{|l|}{ Orden Apodiformes } \\
\hline Sephanoides sephaniodes & Picaflor & $\mathrm{N}$ & OI \\
\hline \multicolumn{4}{|l|}{ Orden Passeriformes } \\
\hline Cinclodes patagonicus & Churrete & I & OI \\
\hline Cinclodes fuscus & Churrete acanelado & I & OI \\
\hline Geositta cunicularia & Minero & I & PV \\
\hline Leptasthenura aegithaloides & Tijeral & I & TA \\
\hline Phleocryptes melanops ${ }^{\mathrm{b}}$ & Trabajador & I & PV \\
\hline Muscisaxicola maclovianus & Dormilona tontita & I & TA \\
\hline Hymenops perspicillatus & Run-run & I & $\mathrm{TA}$ \\
\hline Anairetes parulus & Cachudito & I & TA \\
\hline Xolmis pyrope & Diucón & I & TA \\
\hline Lessonia rufa & Colegial & I & TA \\
\hline Elaenia albiceps & Fío-fío & IF & PV \\
\hline Phytotoma rara & Rara & $\mathrm{HF}$ & OI \\
\hline Tachycineta meyeni & Golondrina chilena & I & TA \\
\hline Pygochelidon cyanoleuca & Golondrina de dorso negro & I & TA \\
\hline Troglodytes aedon & Chercán & I & TA \\
\hline Cistothorus platensis & Chercán de las vegas & I & $\mathrm{TA}$ \\
\hline Turdus falcklandii & Zorzal & IF & TA \\
\hline Mimus thenca & Tenca & IF & TA \\
\hline Anthus correndera & Bailarín chico & I & $\mathrm{TA}$ \\
\hline Sicalis luteola & Chirigüe & G & $\mathrm{TA}$ \\
\hline Zonotrichia capensis & Chincol & G & TA \\
\hline Diuca diuca & Diuca & G & TA \\
\hline Phrygilus alaudinus & Platero & G & OI \\
\hline Phrygilus fruticeti & Yal & $\mathrm{G}$ & PV \\
\hline Sturnella loyca & Loica & IG & TA \\
\hline Molothrus bonariensis & Mirlo & IG & $\mathrm{TA}$ \\
\hline Curaeus curaeus & Tordo & $\mathrm{IH}$ & $\mathrm{TA}$ \\
\hline Agelasticus thilius & Trile & I & TA \\
\hline Sporagra barbata & Jilguero & $\mathrm{G}$ & TA \\
\hline Passer domesticus & Gorrión & GI & $\mathrm{TA}$ \\
\hline
\end{tabular}

${ }^{a}$ Los nombres científicos y comunes se basan en Remsem et al. (2013) y Araya et al. (1995), respectivamente.

${ }^{b}$ Especies registradas después del verano de 2005.

${ }^{c}$ Fuentes: Rottmann 1995, Estades \& Temple 1999, Figueroa et al. 2001.

Recibido: 15.05 .14

Aceptado: 12.03 .16 